\title{
Evaluation of bogie centre bowl friction models in the context of safety against derailment simulation predictions
}

Received: 16 June 2017 / Accepted: 1 February 2018 / Published online: 3 March 2018

(C) The Author(s) 2018. This article is an open access publication

\begin{abstract}
This paper investigates the influence of the models of dry friction suspension component used in the numerical simulation of freight car dynamics on the possible predictions related to safety against derailment. Moreover, the performance of the models is compared on the basis of simulation runtime. Three groups of static and dynamic models of friction are selected as three different approaches which are often utilized in popular multibody simulation tools. The models are used for the centre bowl friction joint between the car body and bogies. The friction torque in the centre bowl affects the rotational resistance of the bogie in motion relative to the car body and consequently influences the interaction forces between the wheels and rails. Simulation results of safety against derailment index obtained from the vehicle curving scenario suggest noticeable differences in predictions of the selected models of friction.
\end{abstract}

Keywords Modelling and simulation · Railway freight car · Friction models · Centre bowl friction

\section{Introduction}

The description of dry friction plays an important role in the modelling and simulation of motion of railway freight vehicles with friction-based suspension components. In most cases, friction in rail vehicle suspensions is modelled as dry Coulomb friction, where the friction force is proportional to the normal reaction. Due to the discontinuity of friction force at close-to-zero velocity, most authors apply regularization to avoid numerical problems in simulation. In the past many studies involving friction dampers in suspensions using this method were conducted. More recent approaches are based on different kinds of non-smooth rheological models which are used in the studies of such authors as Piotrowski, Fergusson, Fröhling, Bogojević, Jönnson, Stichel. Models in this group [1-4] introduce an additional linear spring element in series with a friction slider. The stiffness of the spring can be interpreted as related to the structural tangential flexibility or contact stiffness. A similar idea is used in the bristle interpretation of friction, where the friction is modelled as the average deflection of elastic spring. If the deflection is large enough, the bristles start to slip. Some authors do not describe the details of the friction models used in their simulation studies, which is often the case when the default models provided in the simulation tools are used.

This paper studies the influence of the model of spherical centre bowl friction component used in the simulation of freight car dynamics on the possible predictions related to safety against derailment. The connection between the car body and bogies is designed to have certain properties: it allows the relative rotation between the bogies and car body in curves, transmits the vertical and horizontal forces, assists in maintaining the stability of the vehicle and equalizes load distribution. These properties are implemented differently depending on the type of the rolling stock. In most cases freight bogies have single-stage suspension only, either between the 
bogie frame and bolster, or between the wheelsets and the bogie frame (similar to passenger bogies' primary suspension). The former is more widespread and is based on a diamond-type ("three-piece") bogie with a flat centre plate. The latter type is used mostly in European countries and is known as the standard UIC Y25 bogie. In the case of the Y25 bogie the car body rests on the semi-spherical centre bowl and elastic side bearers without vertical clearance, though the majority of the car body mass rests on the centre bowl. Other features of Y25 bogies are bi-linear vertical suspension stiffness characteristics, and progressive dry friction damping influenced by the vertical load through the angled Lenoir link.

\section{Description of models}

The conventional bogie centre is a bearing made of two parts, namely the upper bogie centre pivot and the lower bogie centre bowl. The upper bogie centre pivot is firmly attached to the underside of the underframe of the vehicle body, and the lower bogie centre bowl is firmly attached to the bolster or crossbar of the bogie frame. The bowl and the corresponding pivot can be flat or spherical. Figure 1 shows the key geometric features of the spherical bogie centre bearing fitted to the Y25 bogie. The bogie centre bearing transmits the vertical load from the vehicle body to the bogie and via its vertical walls also transmits all forces in the horizontal plane. These horizontal forces are mainly the longitudinal traction and braking forces, but also lateral forces such as centrifugal forces arising on curved track. Thus, the standard two-piece bogie centre bearing allows the bogie only one degree of freedom for motions relative to the vehicle body in the horizontal plane, i.e. the rotation about the vertical axis through the geometrical centre of the bogie centre bearing. This rotational freedom allows the bogie frame to align itself radially to the vehicle body on curved track. Due to the presence of the pivot pin bushing in the centre bowl, the contact surface between the pivot and bowl is limited to the ring of the semi-sphere. In the vertical cross section shown in Fig. 1 the arc contour of the ring can be described by the angles $\theta_{0}$ and $\theta_{1}$ measured from the vertical axis. The clearance between the contacting surfaces is negligible.

The magnitude of friction torque in the connection can be calculated from the equation:

$$
M=\int_{\theta_{0}}^{\theta_{1}} R \sin (\theta) \mu \mathrm{d} N
$$

where $\mu$ is the friction coefficient, $R$ radius of the curvature, and $\mathrm{dN}$ the differential normal force in the contact surface given in the form:

$$
\mathrm{d} N=\sigma(\theta) 2 \pi R \sin (\theta) R \mathrm{~d} \theta,
$$

where $\sigma$ is contact pressure function. Calculation of contact pressure distribution can be carried out for two simplified cases. In the first case of sphere-sphere contact it can be assumed that the surfaces are rigid without clearance, the resulting contact pressure is uniform, and its value is equal to the quotient of radial force $F$ and projected contact area $A$. In the second case the contacting bodies are assumed to be elastic with negligible clearance; the contact pressure can be modelled with the function:

$$
\sigma(\theta)=1.5 \frac{F}{A} \cos (\theta) \text {. }
$$
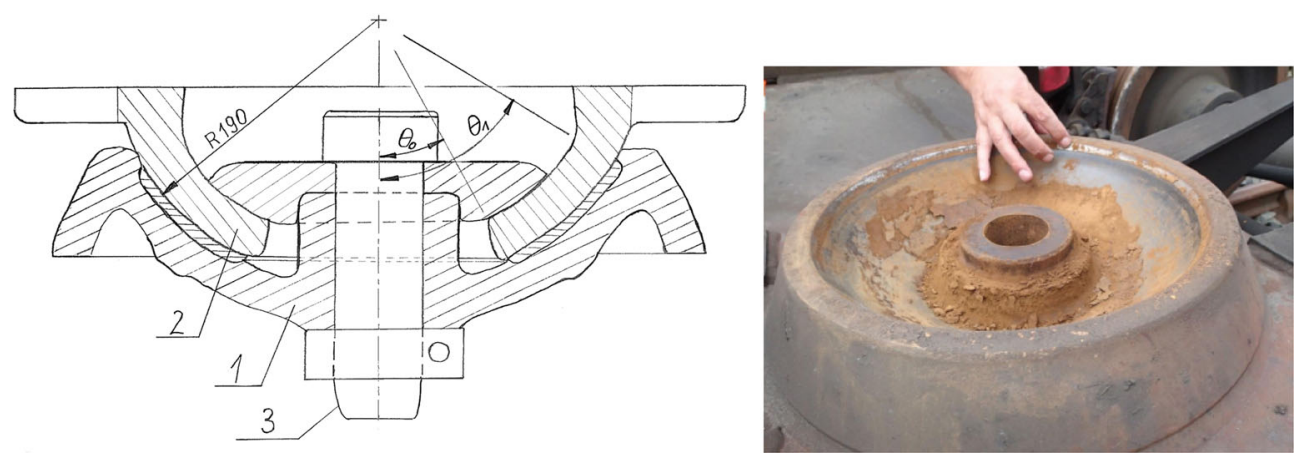

Fig. 1 Left: shape of the centre bowl; 1-bowl, 2-pivot (car body), 3-pivot pin. Right: centre pivot liner worn below the level of steel rim, leading to contact between steel surfaces [5] 


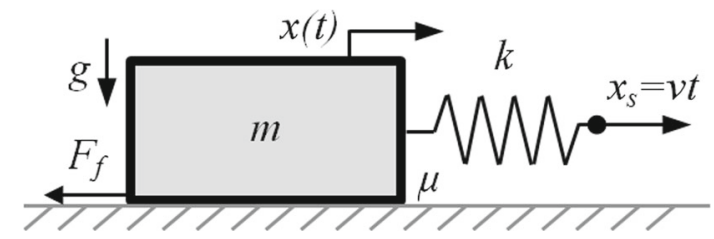

Fig. 2 Model of a tribomechanical system

The resulting value of the maximum frictional torque due to the vertical load $F$ can be denoted by the formula:

$$
M=f \mu F,
$$

where $f$ is a shape coefficient related to the contact geometry and contact pressure distribution. The value of the $f$ coefficient related to the described cases, on the basis of Hertz theory, is in the range of $0.13-0.17$ for steel-steel contact. Additional calculations were made using the finite element method. The obtained value for the $f$ coefficient related to elastic bodies is $17 \%$ smaller (for steel-steel contact with friction). The contact pressure distribution can be affected by other factors as well, e.g. lateral force component due to the centrifugal and centripetal forces. Special cases are not taken into account in this paper.

The calculated friction torque is subsequently used in the model of dry friction in the bogies-car body connection. From the available friction models described in the literature [1-4,7-11,14], three groups of models were selected on the basis of the representation of popular friction models used in rail vehicle simulation. The first model represents the static smooth Coulomb approach, the second model is related to the dynamic non-smooth rheological approach, and the third one is based on the static non-smooth Coulomb approach with a defined region of stiction. There are fundamental differences between the selected models. For the compatibility purposes, the friction models were tested and tuned in an oscillating spring-mass system of one degree of freedom (shown in Fig. 2), which can be used for obtaining the stick-slip behaviour or free vibration damping characteristics, when driving velocity is zero. The individual parameters of the models were tuned in order to obtain compatible macro-characteristics of dynamics behaviour. The system can represent, in some degree of simplification, a wide class of mechanical systems with friction.

The equation which represents the motion is:

$$
m \ddot{x}+k(x-v t)+F_{f}(t, \dot{x}, \ddot{x}, N, \mu)=0 .
$$

Tribomechanical models were implemented in the MATLAB environment, where Eq. (5) is rewritten in the form of a set of first-order differential equations. In the case of the LuGre friction model [8] described in Eq. (8), one of the differential equations is used for describing the internal variable which represents the relative displacement. In the Piotrowski model an additional differential equation is used as well. The Ode45 solver was applied to obtain the numerical solution. The presented example results in Figs. 3, 4 and 5 were obtained for the following parameters: $m=1 \mathrm{~kg}, k=500 \mathrm{~N} / \mathrm{m} ; v=0.01 \mathrm{~m} / \mathrm{s} ; \mu_{d}=0.2 ; \mu_{\mathrm{s}}=1.5 \mu_{d}$.

Improving the Coulomb model by smoothing the discontinuity at zero velocity solves the problem with integration, but the model still lacks the capability to account for stiction. During the stiction phase, there
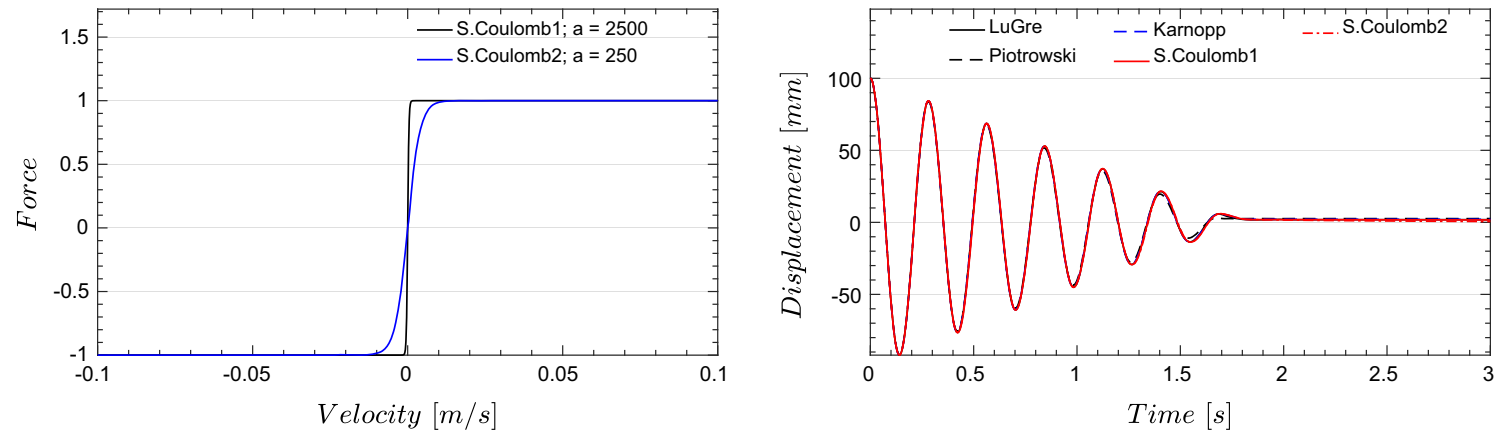

Fig. 3 Smooth Coulomb model characteristics (left); free vibration damping of 1-DOF system with dry friction (right) 


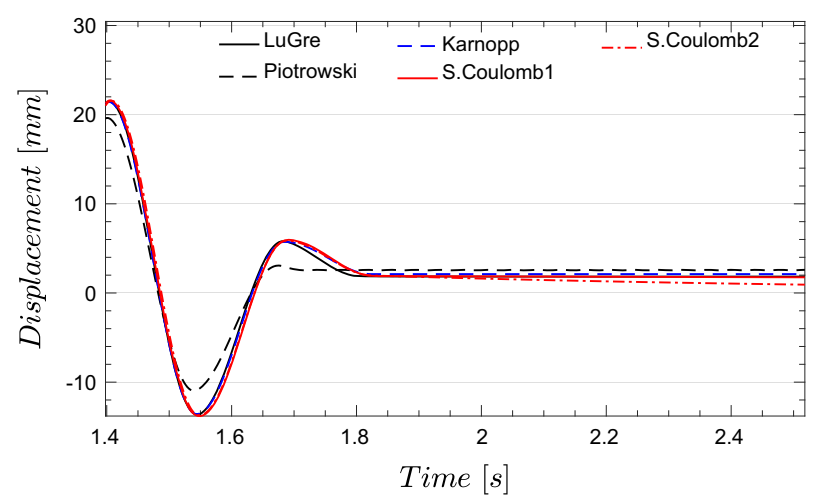

Fig. 4 Zoomed in final phase of free vibration damping when the system should not return to the zero position due to the stiction effect
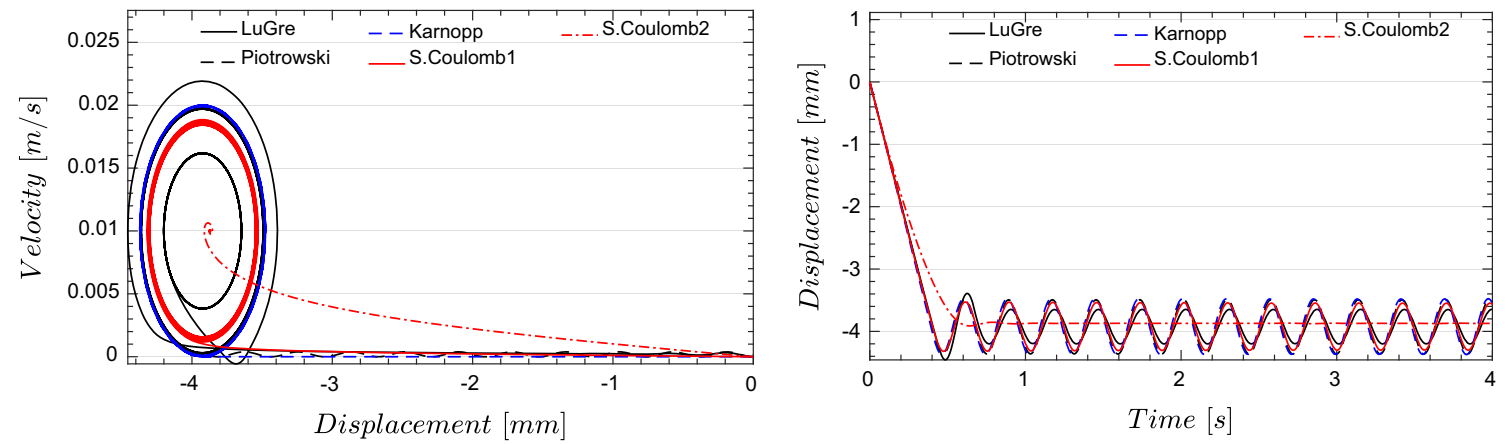

Fig. 5 Comparison of phase plane and time-domain outputs during simulation of stick-slip behaviour

should not be a relative motion between the bodies at the contact point. In this model sticking behaviour is precluded in favour of a low-velocity creep. The smooth Coulomb model is expressed as in Eq. (6).

$$
F_{f}=-F_{\mathrm{d}} \tanh (a v),
$$

where dynamic friction force $F_{d}=\mu_{d} N$ and $v$ is the sliding velocity. Its friction slope characteristics are influenced by the parameter $a$ as shown in Fig. 3. Two different values of this parameter are used, i.e. $a=250$ and $a=2500$.

The Karnopp model $[9,10]$ can better represent stiction behaviour and theoretically is convenient from a computational point of view. It is a partially velocity-based model with an additional dead band introduced where the velocity is considered null due to assumed stiction. Within the dead band, the friction force is proposed to be equal to the external force acting on the object; therefore, the resultant force is zero. The Karnopp friction model can be expressed as in Eq. (7):

$$
F_{f}= \begin{cases}-\min \left(\max \left(-F_{\mathrm{s}}, F_{\mathrm{ext}}\right), F_{\mathrm{s}}\right) & |v| \leq v_{\mathrm{d}} \\ -F_{\mathrm{d}} \operatorname{sgn}(v) & |v|>v_{\mathrm{d}}\end{cases}
$$

where $F_{\text {ext }}$ is the resultant of the external forces, $F_{\mathrm{S}}$ static friction force, and $v_{d}$ dynamic velocity tolerance. The main drawback of the model is the need to know the resultant external force, and this force is not always explicitly given. Moreover, the zero-velocity bands do not reflect the real behaviour of friction.

The LuGre model is a dynamic friction model which is related to the bristle interpretation of friction. Friction is modelled as the average deflection of the elastic spring; if the deflection is large enough the bristles start to slip. The average deflection is an additional internal state which needs to be numerically integrated. The model captures many effects observed in experiments, such as velocity and acceleration dependence, and hysteresis and pre-slip displacement. The basic parameters are (8): bristle (pre-sliding contact) stiffness$\sigma_{0}$, internal damping $-\sigma_{1}$ and viscous damping coefficient $-\sigma_{2}$ (which is assumed to be zero in this study). Remaining parameters are related to the velocity-dependent Stribeck effect. 


$$
\begin{aligned}
F_{f} & =-\sigma_{0} z+\sigma_{1} \dot{z}+\sigma_{2} v \\
\dot{z} & =v\left(1-\frac{\sigma_{0} z}{g(v)} \operatorname{sgn}(v)\right) \\
g(v) & =F_{\mathrm{d}}+\left(F_{\mathrm{S}}-F_{\mathrm{d}}\right) e^{-\left(v / v_{\text {Stribeck }}\right)} .
\end{aligned}
$$

Although more versatile, dynamic friction models are more complex, with more parameters, this makes it more difficult to implement and identify, and also increases computational cost.

In his model [2] Piotrowski uses the element of the Prandtl body composed of the linear spring in series with a slider of the Coulomb friction known as the de Saint Venant element. He formally replaces the action of the friction slider in series with spring by the friction force $F_{f}$ as in Eq. (9). The parameters are as follows: $k$ - contact stiffness and $T_{0}$ - breakout force. Displacements of both ends of the element are denoted by $y$ and $Y$.

$$
\begin{aligned}
\dot{F}_{f} & = \begin{cases}k(\dot{y}-\dot{Y}) & \text { if }\left|F_{f}\right|<T_{0} \\
-[-k(\dot{y}-\dot{Y})]^{+} & \text {if } F_{f}=+T_{0} \\
{[k(\dot{y}-\dot{Y})]^{+}} & \text {if } F_{f}=-T_{0}\end{cases} \\
{[u]^{+} } & = \begin{cases}u & \text { if } u \geq 0 \\
0 & \text { if } u<0 .\end{cases}
\end{aligned}
$$

In this study the boundary values of the selected models' basic parameters were chosen on the basis of mutual matching of their macro-characteristics in two scenarios, i.e. free vibration damping and stick-slip behaviour. Moreover, the LuGre parameter $\sigma_{0}$ is assumed proportional to the normal contact force and the $\sigma_{1}$ parameter proportional to the square root of $\sigma_{0}[11]$. The LuGre $\sigma_{0}$ parameter and Piotrowski $k$ parameter were set to the same value. According to expectations, despite similarities in some regimes, the models generally produce different results at the more fundamental level (Fig. 5).

In order to get a general reference system for testing the validity of models, experimental data were obtained from the test rig shown in Fig. 6. The rig is intended to evaluate body-to-bogie yaw torque which is generated by the passage of the vehicle through curves. It is one of the preliminary methods for testing safety against derailment according to the EN14363 standard. The test is carried out symmetrically in both directions of yaw rotation up to at least a certain body-bogie yaw angle which is calculated on the basis of the vehicle dimensions and minimum curve radius specified for the vehicle. A mean yaw velocity of $1 \%$ s is achieved over at least $75 \%$ of the yaw angle amplitude. Car body-bogie yaw torque is the yaw hysteresis magnitude and results from friction and damping in the system. Due to the lack of yaw stiffness in freight car body-bogie suspensions, the characteristics are mostly flat.

In Fig. 7 a more detailed comparison is presented between the measured and simulated characteristics of the body-bogie yaw friction torque. Simulated results are obtained from the multibody vehicle model. In this model the bogie is rotated in the way that mirrors the test rig behaviour. At the maximum yaw angle of the bogie, the S.Coulomb1-2 models solutions differ from the other solutions, which is related to the zero yaw velocity at this angle and the poor capability to account for stiction.
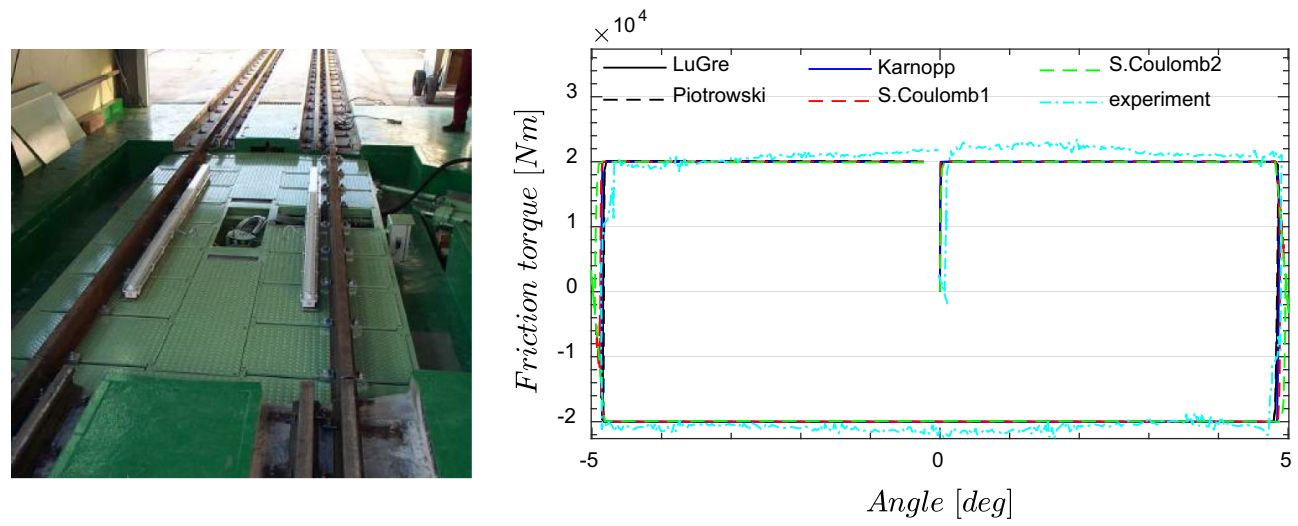

Fig. 6 Measurement of bogie yaw resistance characteristics 


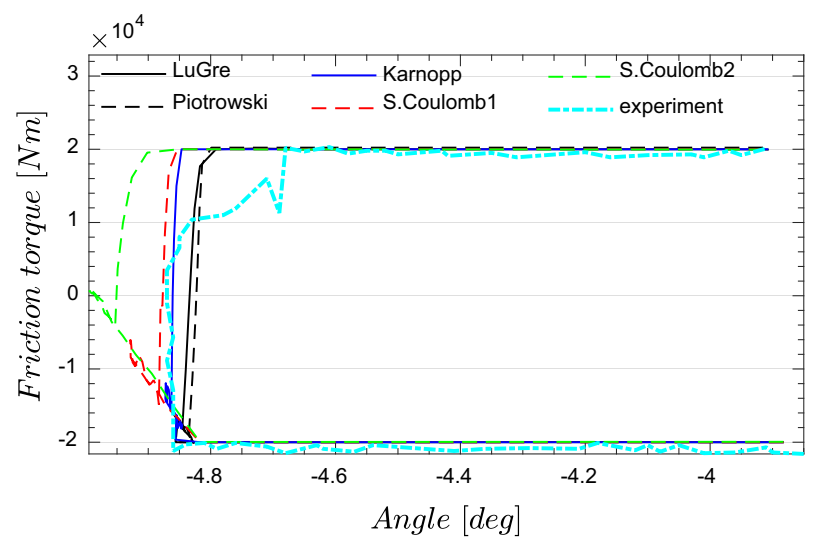

Fig. 7 Zoomed in left part of the friction torque characteristics

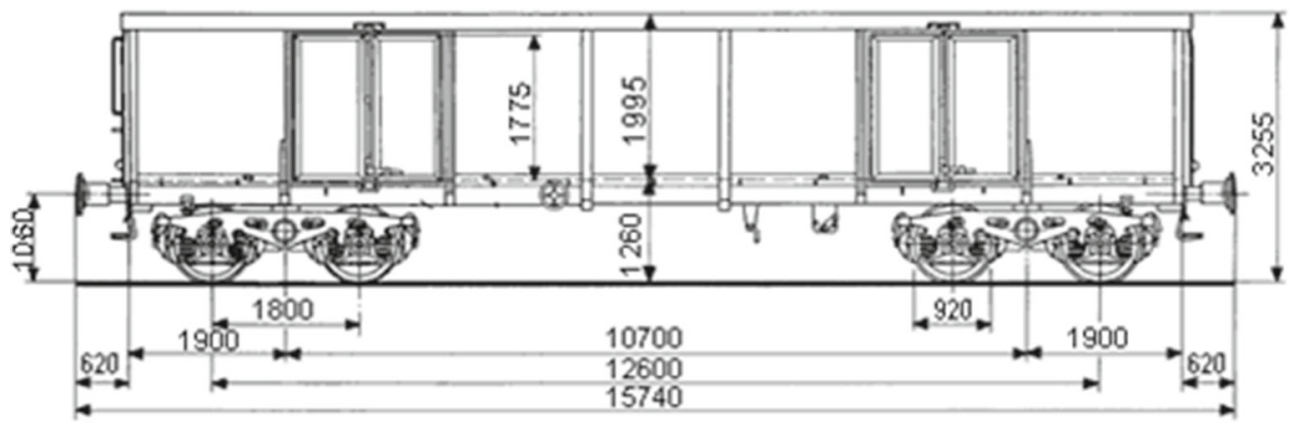

Fig. 8 Geometry of freight wagon Eanos

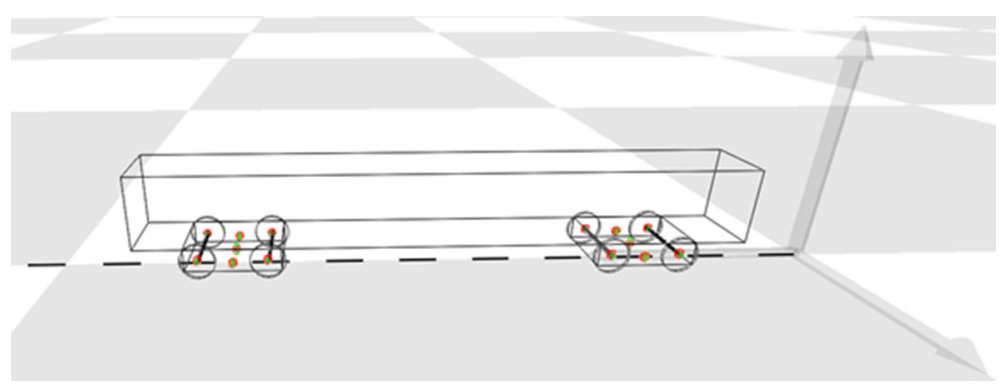

Fig. 9 Representation of the vehicle multibody model

The model of the vehicle is implemented in the author's computer simulation program Simdel [6] in the form of a system of rigid bodies connected with springs and dampers of linear characteristics (Figs. 8, 9). Equations of motion are generated automatically for any given structure of the system. The system of rigid bodies interacts with the track through the higher kinematic pairs of wheels and rails profiles which are provided in the form of coordinates measured on real S1002/UIC60 profiles. The model of contact is based on Kalker's simplified theory and the FASTSIM algorithm. In order to calculate tangential contact forces the algorithm requires such input data as normal contact forces, coefficient of friction (assumed equal to 0.4 on the basis of EN14363:2016 requirements described in the annex B and annex T), length of the semi-axes of the contact ellipses (calculated using Hertz theory), and creep values which are given in the form of relative rigid slip (10):

$$
\left[\begin{array}{c}
r s_{x} \\
r s_{y} \\
r s_{z}
\end{array}\right]=\frac{1}{v u_{x}}\left[\begin{array}{c}
s v_{x} \\
s v_{y} \\
s v_{y} * \sin (\alpha)+s v_{z} * \cos (\alpha)
\end{array}\right],
$$

where $r s_{x}, r s_{y}$ are creepages (relative rigid slip) in the longitudinal and lateral directions, $r s_{z}-$ spin; $\alpha-$ contact angle; $v u$ - speed of the moving reference frame (equal to the vehicle speed); and sv-slip velocity: 


$$
\left[\begin{array}{l}
s v_{x} \\
s v_{y} \\
s v_{z}
\end{array}\right]=\left[\begin{array}{c}
v u_{x} \\
0 \\
0
\end{array}\right]+\left[\begin{array}{c}
0 \\
v r_{y} \\
0
\end{array}\right]+\bar{\omega}_{w} \times \bar{r},
$$

where $\omega_{w}$ is relative angular velocity of the wheelset; $r$-coordinates of the contact point in the reference frame connected to the wheelset mass centre; and vr-relative velocity of the wheelset mass centre (in the moving reference frame).

The equations of motion of the vehicle are generated automatically by the multibody numerical algorithm using the Newton-Euler formalism:

$$
\begin{aligned}
& m_{i} \dot{v}_{i}=F_{i}, \\
& J_{i} \dot{\omega}_{i}+\omega_{i} \times J_{i} \omega_{i}=T_{i}, \\
& F_{i}=f_{i-1}-f_{i+1}+m_{i} g, \\
& T_{i}=t_{i-1}-t_{i+1}+d_{i} \times f_{i-1}-r_{i} \times f_{i+1},
\end{aligned}
$$

where $f, t$ are forces and torques from precedent and subsequent bodies acting in joints; $F, T$-resultant forces and torques acting on the mass centre; and $d, r$-distances between the joint and the mass centre of the body. Selected equations for the individual bogie are given below:

$$
\begin{aligned}
F_{z b i} & =V_{c i}+\left(V_{s b i 1}+V_{s b i 2}\right)-\left(V_{w i 1}+V_{w i 2}\right)+m_{b} g, \\
T_{\psi b i} & =T_{c i}+b\left(F_{f s b i 1}^{x}-F_{f s b i 2}^{x}\right)-\left(T_{w i 1}^{z}+T_{w i 2}^{z}\right)-a\left(H_{w i 1}+H_{w i 2}\right), \\
T_{\chi b i} & =b\left(V_{s b i 1}+V_{s b i 2}\right)+h\left(H_{w i 1}+H_{w i 2}\right)-\left(T_{w i 1}^{x}+T_{w i 2}^{x}\right),
\end{aligned}
$$

where $F_{z b i}, T_{\psi b i}, T_{\chi b i}$-resultant vertical force, yaw and roll torques acting on the $i$ th bogie, $F_{f s b i 1,2}^{x}-$ longitudinal friction forces between the side bearers and the car body, $T_{c i}$-friction torque in the centre bowl, $V_{c i}, V_{s b i 1,2}$-vertical suspension force in the centre bowl and in the side bearers, $V_{w i 1,2}, H_{w i 1,2}$-vertical and horizontal primary suspension forces, $T_{w i 1,2}^{x}, T_{w i 1,2}^{z}$-roll and yaw primary suspension torques on wheelsets, $a$-longitudinal semi-spacing of wheelsets (bogie semi-wheelbase), $b$-lateral semi-spacing of side bearers, $h$-vertical spacing between the wheelset end and the bogie centre of gravity, $m_{\mathrm{b}} g$ - bogie frame weight.

In Tables 1 and 2 the basic parameters of the freight car model are reported.

\section{Simulation scenarios}

During the curve negotiation the bogies must rotate relative to the car body. This rotation is restrained by the friction torque generated at the surface of the centre bowl and to some degree at the side bearings. If the vehicle has secondary suspensions, their stiffness could contribute to rotational resistance as well [12]. To overcome the resisting torque an adequate external turning torque must be generated from the interaction forces between

Table 1 Masses and inertias data

\begin{tabular}{lcccr}
\hline Inertia & $\begin{array}{l}\text { Mass } \\
(\mathrm{kg})\end{array}$ & $\begin{array}{l}\text { Roll inertia } \\
\left(\mathrm{kgm}^{2}\right)\end{array}$ & $\begin{array}{l}\text { Pitch inertia } \\
\left(\mathrm{kgm}^{2}\right)\end{array}$ & $\begin{array}{c}\text { Yaw inertia } \\
\left(\mathrm{kgm}^{2}\right)\end{array}$ \\
\hline Body & & & & \\
$\quad$ Car body & 70,000 & 59,383 & 928,320 & 953,980 \\
Bogie frame & 2070 & 1400 & 2100 & 2400 \\
Wheelset & 2000 & 1200 & 200 & 1200 \\
\hline
\end{tabular}

Table 2 Primary suspension characteristics

\begin{tabular}{lllr}
\hline Suspensions & Longitudinal $(\mathrm{kN} / \mathrm{m})$ & Lateral $(\mathrm{kN} / \mathrm{m})$ & Vertical $(\mathrm{kN} / \mathrm{m})$ \\
\hline Primary & 4000 & 4500 & 3984 \\
Primary inner spring & - & - & 6476 \\
Centre pivot-bowl & 10,000 & 10,000 & 10,000 \\
Side bearers & - & 350 & 500 \\
\hline
\end{tabular}


the rails and wheels of the bogie. These interaction forces are usually described as lateral and longitudinal components. Increased contact forces are closely connected to the undesirable effects such as wear and higher probability of derailment.

In simulation scenarios it was assumed that the resisting torque depends on the load level of the car body and lubrication of the centre bowl. Railway operators use different techniques and materials to control the centre bowl friction through lubrications such as liquid, grease, different types of polymer liners, or use no lubrication at all. The resulting coefficient of friction can be in a range from 0.1 (very well lubricated) to above 0.6 (which is considered as the result of a defective centre bowl) [13]. In the simulation, the friction coefficient value of 0.6 is used, as well as axle load level of 20 t/axle related to the loaded car body.

Simulation studies comprise a ride through a smooth right circular arc with radius of 500 or $1000 \mathrm{~m}$ without gauge widening (constant gauge $1435 \mathrm{~mm}$ ) with cant $h=0.1 \mathrm{~m}$. Transition curves of length $75 \mathrm{~m}$ are present between the straight sections and the arc. The length of the regular arc section is $120 \mathrm{~m}$; it starts at $125 \mathrm{~m}$ and ends at $245 \mathrm{~m}$. The vehicle rides with a constant speed of $80 \mathrm{~km} / \mathrm{h}$. Simulation studies are carried out to investigate the safety against derailment according to the Nadal criterion with reference to the rotational resistance of the bogie due to friction in the centre bowl. Despite some drawbacks, the Nadal criterion is commonly used to evaluate proneness to a derailment situation of a single wheel. The admissible value of the $Y / Q$ index can be calculated using Nadal's formula:

$$
\left(\frac{Y}{Q}\right)_{\mathrm{adm}}=\frac{\tan (\alpha)-\mu}{1+\mu * \tan (\alpha)},
$$

where $Y$ and $Q$ refer to the lateral and vertical forces acting between the rail and wheel, $\alpha$ is the angle made when the wheel flange is in contact with the rail edge, and $\mu$ is the coefficient of friction between the wheel and the rail.

\section{Comparison of simulation results}

Figure 10 shows the leading bogie yaw angle relative to the car body, whereas Fig. 11 shows the relative yaw velocity on the same bogie while running through a 500-m curve at $80 \mathrm{~km} / \mathrm{h}$ on a smooth and rigid track. This speed is above the balancing speed for the curve. The yaw velocity of the bogie is a similar order of magnitude for all the models. The models differ in reproducing the stiction effect, which is shown in Fig. 10, particularly in the arc section and after the wagon exits the curve. Due to the stiction, the centre bowl does not necessarily return to its original position. If there is insufficient moment being generated between the wheels and the track to overcome the friction at the centre bowl, the bogie can run in a misaligned position.

All the results are obtained for the leading outer wheel of the vehicle. Figures 12 and 13 show a comparison between the $Y / Q$ values produced by simulation of the freight vehicle with different mathematical models of friction in the centre bowl.

During the negotiation of a medium curve with radius $500 \mathrm{~m}$ (Fig. 12) all the models with the exception of S.Coulomb2 give similar results at the transition curve sections, yet at the regular arc section and after exiting

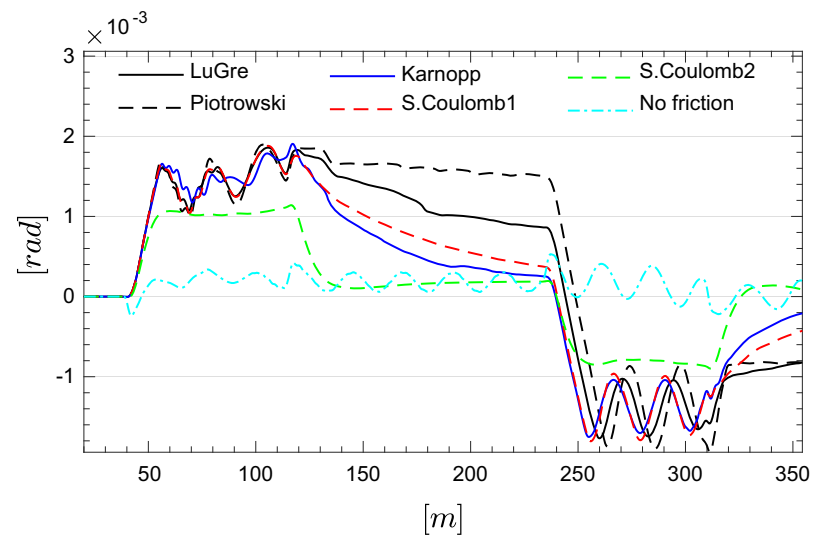

Fig. 10 Leading bogie yaw angle relative to the car body yaw angle. Loaded wagon, centre bowl friction coefficient $\mu=0.6$, arc radius $R=500 \mathrm{~m}$, cant $h=0.1 \mathrm{~m}$, velocity $80 \mathrm{~km} / \mathrm{h}$ 


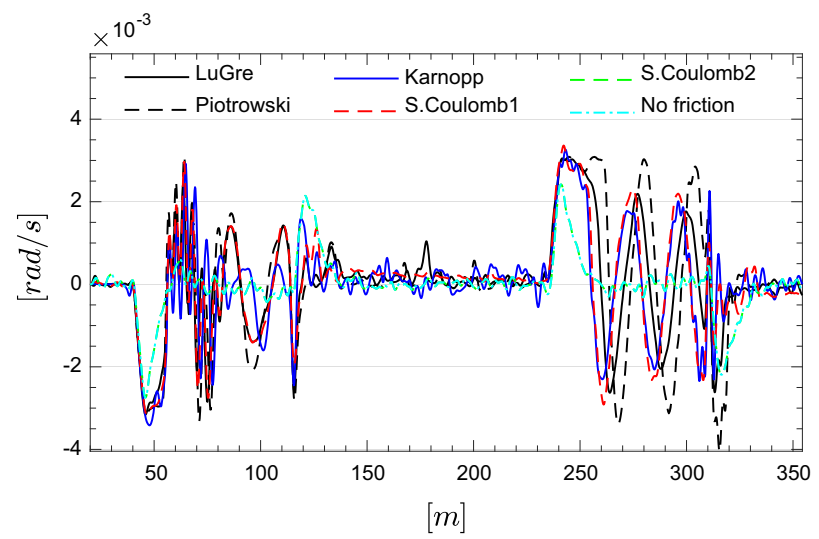

Fig. 11 Relative yaw velocity between the leading bogie and the car body

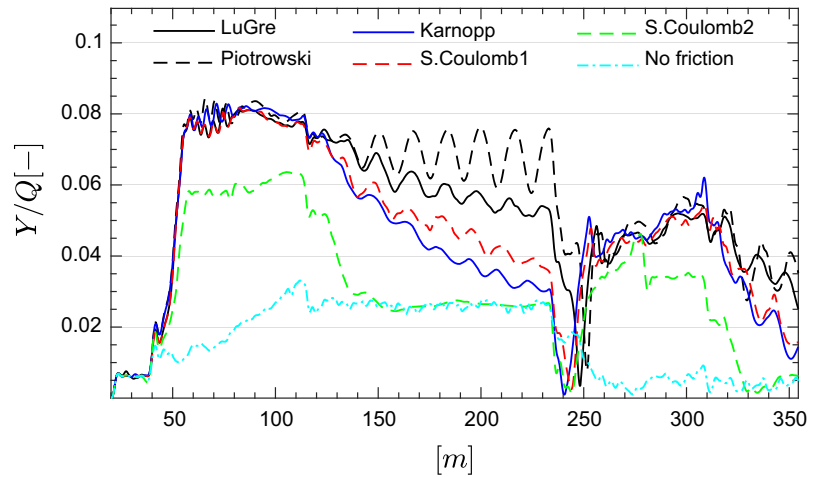

Fig. 12 Comparison of the $Y / Q$ values for different friction models. Loaded wagon, centre bowl friction coefficient $\mu=0.6$, arc radius $R=500 \mathrm{~m}$, cant $h=0.1 \mathrm{~m}$, velocity $80 \mathrm{~km} / \mathrm{h}$

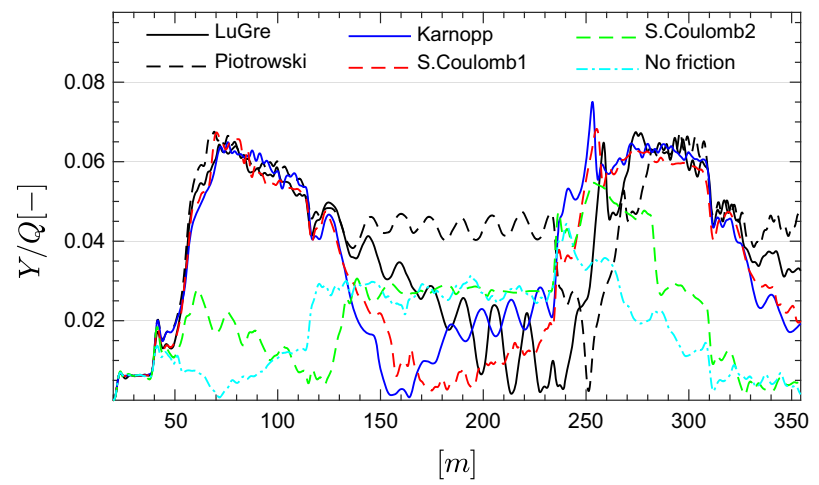

Fig. 13 Comparison of the $Y / Q$ values for different friction models. Loaded wagon, centre bowl friction coefficient $\mu=0.6$, arc radius $R=1000 \mathrm{~m}$, cant $h=0.1 \mathrm{~m}$, velocity $80 \mathrm{~km} / \mathrm{h}$

the curve the results differ. This is due to the different properties of the models in the region of low velocity, the quasi-static motion of the vehicle, as well as the different capabilities of the stiction effect representation. Moreover, the individual settings of the parameters in Karnopp and S.Coulomb models strongly influence the balance between the robustness of the solutions and the calculation speed. The differences are more visible in Fig. 13, which shows the results of the simulation using a curve radius of $1000 \mathrm{~m}$.

In Figs. 14 and 15 the results are shown for the simulation using the same track configuration as in Figs. 12 and 13, but the track geometry has lateral irregularities. Due to the excitation, the vehicle with friction-damped suspension elements tends to produce continuous oscillatory suspension forces which appear most of the time to reach or exceed a value equal to the friction level. Therefore, the friction dampers are sliding for a large 

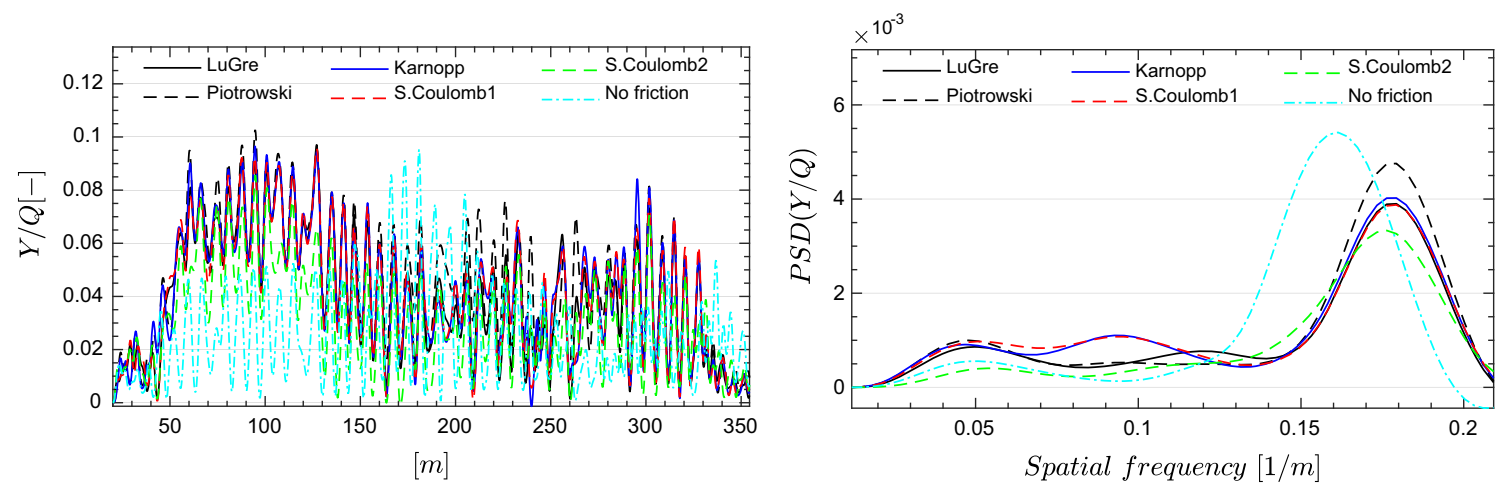

Fig. 14 Comparison of the $Y / Q$ values for different friction models in the space and frequency domains. Track with lateral irregularities. Loaded wagon, centre bowl friction coefficient $\mu=0.6$, arc radius $R=500 \mathrm{~m}$, cant $h=0.1 \mathrm{~m}$, velocity $80 \mathrm{~km} / \mathrm{h}$
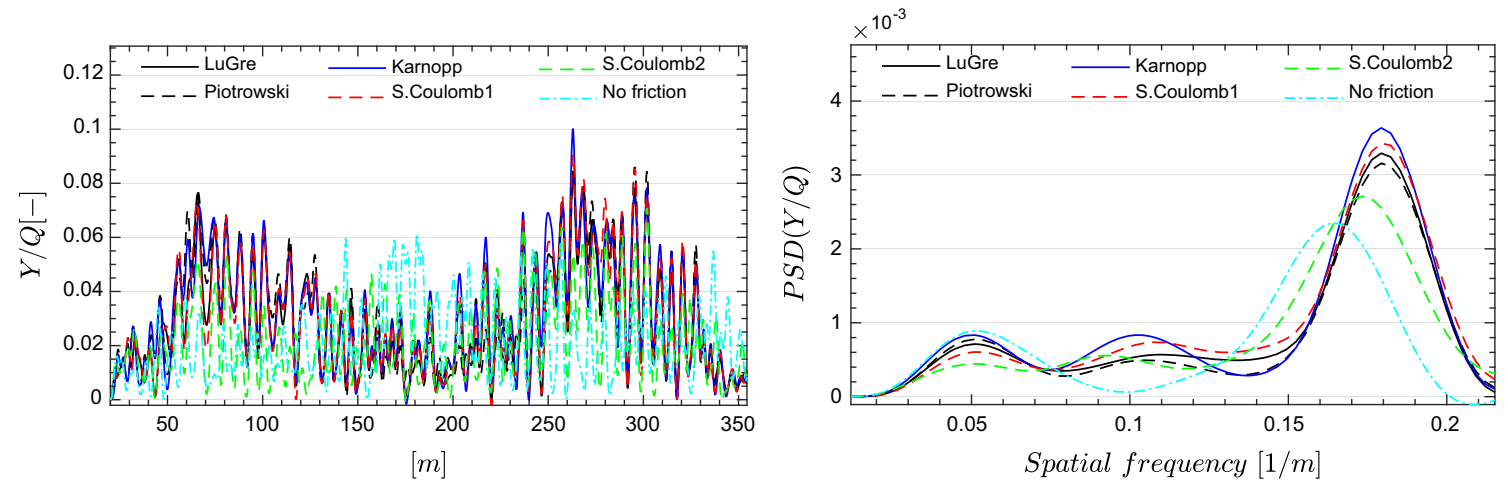

Fig. 15 Comparison of the $Y / Q$ values for different friction models in the space and frequency domains. Track with lateral irregularities. Loaded wagon, centre bowl friction coefficient $\mu=0.6$, arc radius $R=1000 \mathrm{~m}$, cant $h=0.1 \mathrm{~m}$, velocity $80 \mathrm{~km} / \mathrm{h}$

Table 3 Simulation runtime index (the lesser is better)

\begin{tabular}{lcc}
\hline Model & Smooth track & Track with irregularities \\
\hline LuGre & 8 & 15 \\
Piotrowski & 25 & 28 \\
Karnopp & 58 & 155 \\
Smooth Coulomb1 & 22 & 38 \\
Smooth Coulomb2 & 6 & 19 \\
\hline
\end{tabular}

proportion of the time. In such conditions the solution differences between the static and dynamic dry friction models are less evident. The solutions presented in the frequency domain show few differences between the models. The differences are, however, in the simulation runtime, which is generally longer. According to Table 3 , the biggest impact is to the Karnopp model, and the Piotrowski model is least influenced. The LuGre model offers the best overall performance in the selected simulation scenarios.

The performance of the friction models being used in the vehicle-track system in the connection between the car body and the bogies is compared on the basis of the simulation runtime. Obtained results are shown in Table 3. Three numerical methods were used in the simulations, i.e. Adams-Moulton, backward differentiation formula, and Runge-Kutta $(4,5)$. The first one was the main method, and the other methods were used for comparison purposes.

\section{Conclusions}

In the studied case of the railway freight vehicle curving behaviour in the context of safety against derailment, the selection of the bogie centre bowl friction model had a visible impact on the simulation results. For the 
purpose of the calculation of the safety indexes in close-to-quasi-static conditions, using the simple regularized Coulomb model is difficult to provide satisfactory results due to the poor capability to account for stiction, and in the case of the Karnopp model there were difficulties in the implementation. Theoretically, the Karnopp model can better represent stiction behaviour in comparison with the smooth Coulomb1 model, which is also confirmed in the comparison with the experimental data from the test rig. However, in the simulation of the railway vehicle curving it is evident that the retention of the bogie yaw angle (Fig. 10) is smaller in the case of the Karnopp model. The assumed explanation is that the bogie yaw velocity (Fig. 11, track section 130-245 m) oscillates more in the case of the Karnopp model due to the non-smooth transitions between the stiction (dead band) and slip areas, and therefore, the friction changes its sign more often and its effect is less directional on average. Changing the value of the dead-band parameter does not change this behaviour considerably. The potential effect is noticed if the signum function is replaced with the smooth function. A similar approach is taken in [14] wherein the model is also using an external force and the stick-slip transition is regularized with a hyperbolic secant function. The overall performance of these models based on the simulation runtime and robustness of the solutions is lower than the other presented models, such as LuGre and Piotrowski.

In more dynamic conditions arising from track geometry irregularities, the differences among the simulation results are related more to the simulation runtime. Eventually, the selection of a friction model depends on its intended application. Systems operating mainly in the sliding regime will benefit less from a dynamic friction model compared to a system operating at low velocity, or where velocity changes its sign often.

Open Access This article is distributed under the terms of the Creative Commons Attribution 4.0 International License (http:// creativecommons.org/licenses/by/4.0/), which permits unrestricted use, distribution, and reproduction in any medium, provided you give appropriate credit to the original author(s) and the source, provide a link to the Creative Commons license, and indicate if changes were made.

\section{References}

1. Bruni, S., Vinolas, J., Berg, M., Polach, O., Stichel, S.: Modelling of suspension components in a rail vehicle dynamics context. Vehicle Syst. Dyn. Int. J. Veh. Mech. Mobil. 49(7), 1021-1072 (2011). https://doi.org/10.1080/00423114.2011. 586430

2. Piotrowski, J.: Model of the UIC link suspension for freight wagons. Arch. Appl. Mech. 73(517), 517-532 (2003). https:// doi.org/10.1007/s00419-003-0305-6

3. Fergusson, S.N., Fröhling, R.D., Klopper, H.: Minimising wheel wear by optimising the primary suspension stiffness and centre plate friction of self-steering bogies. Veh. Syst. Dyn. Int. J. Veh. Mech. Mobil. 46(S1), 457-468 (2008). https://doi. org/10.1080/00423110801993094

4. Bogojević, N., Jönsson, P., Stichel, S.: Iron ore transportation wagon with three-piece bogies-simulation model and validation. In Proceedings of the 7th international scientific conference Heavy Machinery (HM2011), Faculty of Mechanical Engineering, Kraljevo, ISBN 978-86-82631-58-3, pp. 39-44 (2011)

5. Clark, G.: Urgent Safety Advice, Incident report no 0776, RAIB, Department for Transport, UK (2015)

6. Opala, M., Melnik, R.: Ocena zgodności modeli symulacyjnych dynamiki pojazdów szynowych na podstawie testów referencyjnych (Concordance assessment of the rail vehicles' dynamic models based on the benchmark tests). Czasopismo Logistyka, vol. 4/2015, ISSN 1231-5478 (2015)

7. Pennestri, E., Rossi, V., Salvini, P., Valentini, P.: Review and comparison of dry friction force models. Nonlinear Dyn 83(4), 1785-1801 (2016)

8. de Canudas, Wit C., Olsson, H., Astrom, K.J., Lischinsky, P.: A new model for control of systems with friction. IEEE Trans. Autom. Control 40(3), 419-425 (1995)

9. Karnopp, D.: Computer simulation of slip-stick friction in mechanical dynamic systems. J. Dyn. Syst. Measur. Control 107(1), 100-103 (1985)

10. Bicakci, S., et al.: Optimizing Karnopp friction model parameters of a pendulum using RSM. European Journal of Control (2014). https://doi.org/10.1016/j.ejcon.2014.04.001

11. Liu, B.X., Nie, S.H.: Dynamic Parameters Identification of LuGre Friction Model Based on Chain Code Technique. The 14th IFToMM World Congress (2015), 67/IFToMM.14TH.WC.PS4.009

12. Shi, Huai-long, Ping-bo, Wu, Luo, Ren, Guo, Jin-ying: Calculation and laboratory testing of the rotation resistance of a bogie. J. Rail Rapid Transit 229(2), 210-219 (2015). https://doi.org/10.1177/0954409713508110

13. Wu, H., Robeda, J.: Effects of bogie centre plate lubrication on vehicle curving and lateral stability. Veh. Syst. Dyn. Suppl. 41, 292-302 (2004)

14. Xia, F., True, H.: On the dynamics of the three-piece-freight truck. RTD-Vol. 25, In Proceedings of the 2003 IEEE/ASME Joint Rail Conference, IEEE, Chicago Ill, pp. 149-159, American Society of Mechanical Engineers (2003) 